\title{
Assessment of Genetic Shielding for Adenovirus Vectors
}

\author{
Susan J. Hedley ${ }^{1}$, Aleksandr Krendelshchikov ${ }^{1}$, Myung-Hee Kim ${ }^{1}$, Jian $\mathrm{Chen}^{2}$, Hui-Chen Hsu ${ }^{2}$, \\ John D. Mountz ${ }^{2}$, David T. Curiel ${ }^{3}$ and Imre Kovesdi ${ }^{1 * *}$
}

\begin{abstract}
${ }^{I}$ VectorLogics, Inc., Birmingham, AL, 35294, USA; ${ }^{2}$ Department of Medicine, Division of Clinical Immunology and Rheumatology at the University of Alabama at Birmingham, AL 35294, USA; ${ }^{3}$ Division of Human Gene Therapy, Departments of Medicine, Pathology, and Surgery, and the Gene Therapy Center at the University of Alabama at Birmingham, AL 35294, USA
\end{abstract}

\begin{abstract}
Development of adenovirus (Ad) vectors in the clinical context has highlighted that vector efficacy may be limited by the host humoral response due to pre-existing titers of neutralizing antibodies against the vector itself in humans. Further, multiple dosing of Ad vectors based on serotype 5 would be limited. Current immune evasion strategies being investigated by other laboratories are only applicable to non-replicating vectors. Therefore we have proposed genetic shielding as an alternate that would be applicable to both non-replicating and conditionally replicating Ad vectors. Genetic shielding would encapsulate fusion of a self-protein to Ad minor capsid protein, pIX, as a means to cloak immunogenic capsid epitopes and prevent neutralization of Ad vectors through Ad specific antibodies. In the development of a suitably shielded Ad vector we choose several self-proteins that we attempted to fuse to pIX. We have also used an indirect method to conjugate albumin to the capsid through an albumin binding domain fused to pIX. Despite attaining novel pIX modified Ad vectors we found that none of the pIX attached molecules in this study prevented neutralizing antibodies from halting gene transfer.
\end{abstract}

\section{INTRODUCTION}

Within the human population there are high titers of preexisting neutralizing antibodies against adenovirus serotypes 2 and $5[1,2]$ due to the general exposure to Ads. Therefore it has been proposed that efficacy of Ad vectors in the clinic would be initially limited and effective repeat administration of Ad vectors will be hindered by strong neutralizing antibody response to the vector. Thus far skeletal muscle was one of the few tissues where repeat Ad vector administration was successfully demonstrated [3]. However, the success of this procedure was highly dependent on the initial dose of Ad used in the experiment and therefore, it is still expected that repeat dosing in human will be problematic. With respect to conditionally replicative Ad vectors (CRAd) data from clinical trials were suggestive of limited efficacy due to strong innate immune responses [4]. Therefore development of non-replicating Ads, or CRAds, capable of evading the humoral response would improve clinical utility of these vectors.

There are two major efforts in an attempt to circumvent this issue with Ad vectors; (1) development of Ad vectors from different serotypes and (2) biochemical coating of the Ad vector. With respect to utilizing different serotypes, Ad11 and Ad35, to which the human population has a lower prevalence of neutralizing antibodies, have been investigated as alternative vectors to those of Ad5 [1, 5-7]. However, other human serotypes such as Ad6 [8], Ad19a [9, 10] and Ad41 [11], and adenovirus serotypes from other species are

*Address correspondence to this author at the VectorLogics, Inc. 550 11th Street South, Birmingham, Alabama 35294, USA; Tel: (205) 933-8378; Fax: (205) 933-5836; E-mail: ikovesdi@ vectorlogics.com also being considered [12-16] as means to overcome preexisting Ad5 immunity. It is also likely that the development of humoral and cellular immune responses against these alternate serotypes will eventually occur. Another issue to consider is the different intrinsic properties of the various novel Ad serotype vectors such as the use of different cellular receptors for different serotype vector groups [17]. In this regard, it has been shown recently, that Ad vectors based on serotype B (like Ad35 and Ad11) binding to their cognate receptor $\mathrm{CD} 46$ down regulate immune responses [18] which has yet to be fully determined as a desirable attribute.

Biochemical modification of the Ad vector potentially provides shielding from the immune response through molecules covering the capsid. One established methodology is the conjugation of functional PEG to free lysine groups on the adenoviral capsid that enable Ad vectors to avoid neutralizing antibodies, in vitro or in vivo or limit the innate response [19-25]. In addition slower clearance rates of Ad from the blood have been demonstrated [26, 27]. Despite major improvements this method has several potential limitations including the ablation of the Ad receptor (CAR) specificity resulting in low levels of infection $[19,20,23$, 25]. Furthermore the heterogeneity of composition will confound potency in batch-to-batch production. These issues alone represent significant problems with respect to scale up and regulatory approval. Finally this method is suitable to protect only non-replicating Ad vectors as in the context of CRAd physiology the shielding molecule is lost from the progeny vectors.

We have previously proposed a concept based upon genetic shielding as an alternate and potentially much simpler method to cloak Ad vectors [28]. The concept was to utilize a self-protein fused to an adenovirus capsid protein to pro- 
vide a shield masking the immunogenic epitopes of the Ad vector capsid. Importantly it would be broadly applicable to both non-replicating vectors and conditionally replicating vectors. To prove this concept, we determined that the minor capsid protein, pIX, would be a suitable anchorage position for the shielding molecule. This is due to the large body of work indicating that carboxy terminus of pIX can be fused to an array of molecules including polylysine [29], the biotin acceptor peptide (BAP) [30] and larger complex proteins including various fluorescent proteins, HSV thymidine kinase (TK) and TK fusions [31-36]. In these studies it was demonstrated that virus viability was not significantly affected and that the pIX-fused molecules retained functionality. Furthermore, pIX is a triplet present at 80 locales, thus allowing for a defined number of shielding molecules to be included into the capsid, and allowing potentially for significant coverage of the capsid. However, the shielding protein of choice would need to embody several important characteristics, namely that it is a self-protein and has sufficient size to cover immunodominant Ad capsid epitopes. In this regard albumin could be a good choice as it is a large monomoeric non-gylcosylate polypeptide with wide in vivo distribution, long half-life and lack of substantial immunogenicity [37]. It has been fused to human growth hormone-recombinant human serum albumin (rHSA) [38], recombinant granulocyte colony stimulating factor-rHSA [39], and serum albuminCD4 genetic conjugate [40] to enhance circulating half-life and improve stability for therapeutic applications. In addition it has a safe record in clinical practice, as Albuferon ${ }^{\mathrm{TM}}$ (albumin-interferon alpha) [41] has completed phase II clinical trials [42].

To examine our concept of genetic shielding we decided to use two different strategies with human albumin (hALB), direct fusion to pIX for capsid incorporation into the capsid and indirect linkage through conjugation methods (Fig. 1). In addition to using hALB directly fused to pIX we decided to utilize another self-protein, alpha-1-antitrypsin (A1A), in parallel for direct incorporation. Although it is not as large as hALB, A1A is of a comparable size to HSV-TK, which has been successfully fused to pIX in replicating Ad vectors [31]. A1A is a serine proteinase inhibtitor with a primary function to inhibit the enzyme neutrophil elastase. We were concerned using an active enzyme and therefore mutated A1A through site directed mutagenesis to obtain A1A (P1-P1') which retains normal folding but results in ablation of enzyme function [43]. In an alternate approach to attempt to prove the concept of shielding with molecules located at the pIX locale, a second strategy was used that would involve conjugating hALB to the capsid through linkage to a binding motif (Fig. 1). Bacteria express surface proteins that interact with human extracellular proteins including several natural albumin binding proteins that are well characterized, such as motifs in Protein G and Protein PAB [44]. Pathogens use these proteins to avoid detection by the human immune system. The small ligand BAP has been successfully incorporated into pIX and been shown to conjugate to appropriately labeled ligands [30], validating our approach and therefore we decided to incorporate the albumin binding domain 3 from Streptococcal Protein G (ABD) [44, 45] into pIX to be utilized as a docking site for albumin.

\section{MATERIALS AND METHODOLGY}

\section{Cell Line Culture}

HEK 293 cells and A549 cells were purchased from ATCC (Manassas, VA). All cells were propagated in Dulbecco's modified Eagle's medium (DMEM)/F-12 medium with $10 \%$ FBS, $2 \mathrm{mM}$ glutamine, $100 \mathrm{U} / \mathrm{ml}$ penicillin, and $100 \mu \mathrm{g} / \mathrm{ml}$ streptomycin. FBS was purchased from HyClone

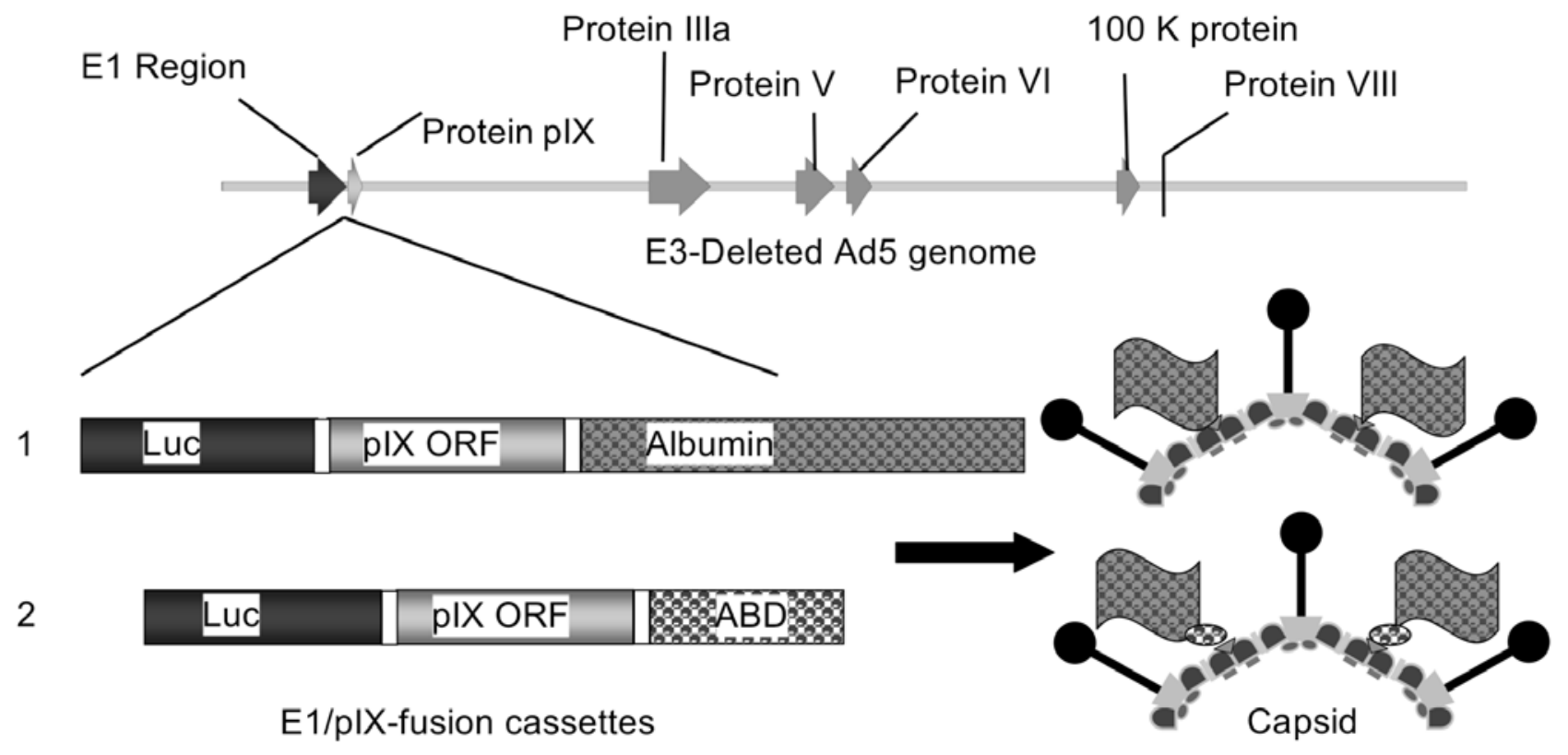

Fig. (1). Concept of direct and indirect incorporation of genetic shield proteins into Ad vector capsid. The E1/pIX fusion cassettes illustrate the concept of (1) direct incorporation of albumin through genetic fusion to the pIX molecule, and (2) indirect incorporation of albumin through conjugation to an albumin binding domain (ABD) genetically fused to the pIX molecule. The E1/pIX fusion cassettes are recombined into the Ad genome at the position shown. In the capsid configuration, the ABD domain is shown as a spotted circle and albumin is shown as a spotted flag. 
(Logan, UT), and media and supplements were from Mediatech (Herndon, VA). All cells were propagated at $37^{\circ} \mathrm{C}$ in a $5 \% \mathrm{CO}_{2}$ atmosphere.

\section{Capsid Modified Adenoviral Vectors}

To generate the proposed shielded Ad vectors in this study, we used pIX-modified vectors which were based on the pSILucIXFlag-NheI shuttle vector [29] (or linker variants, $45 \AA$ and $75 \AA$ based on those described by Vellinga and coworkers [46]). To fuse proposed shield proteins to the pIX capsid protein, the shield proteins were cloned into the NheI sites of the shuttle vectors as follows. The cDNA of human alpha 1 antitrypsin (A1A) and human preproalbumin are commercially available in the plasmids pCMV6-XL6 and pCMV4-XL6 respectively (Origene, Rockville, MD). To generate a PCR fragment of A1A with NheI ends the following primers (IDT, Coralville, IA) were used sense 5' -tgacaagctagccgaggatccccagggagat-3' and anti-sense 5' -gcgcatggctagcttattttgggtgggat-3' and to generate the mature form of hALB with NheI ends the following primers were used sense 5' - gacaagctagccgatgcacacaa-3' and antisense 5'-ggatggctagcttataagcctaag-3' (NheI sites underlined). PCR fragments once purified were NheI digested and cloned into the shuttle vectors. Site directed mutagenesis of A1A to generate Met358Pro and Ser359Pro (A1A(P1-P1')) took place in over two rounds using the following primers (altered nucleotides in bold), Step 1: sense 5'-ggccatacccatgtctatccca ccagaggtcaagttcaacaaac-3' and anti-sense 5' - gtttgttgaacttga cctctggtgggatagacatgggtatggcc-3' and Step 2: sense 5' -gttttta gaggccataccaccgectatccaccagaggtcaag-3' and anti-sense 5' -cttgacctctggtgggataggcggtggtatggcctctaaaac-3'. The two steps were performed using the QuikChange Site Directed Mutagenesis kit following the manufacturer's protocol (Stratagene, La Jolla, CA). As a control for pIX modification and viral rescue we also made an AdLuc vector containing a mutated TK gene (sr39TK) as this is a known successful pIX modification [31]. For sr39TK the NheI fragment was out of pShuttle.wt.E1-pIX-flag-TK [31]. The albumin binding domain 3 (ABD) from streptococcal Protein G (46 amino acid) [44, 45] (Genbank X06173) was generated using two commercially synthesized single-stranded oligonucleotides (IDT), one starting at the 5' end, the other starting at the 3 ' end, with an overlapping central 15 nucleotide region. The oligonucleotides were annealed and extended, using Pfu Hifidelity TAQ (Stratagene), to form a double stranded (ds) cDNA of ABD with NheI restriction sites at both the 5' and 3' ends. The ds cDNA was digested with NheI to allow for subcloning into the pSILuc shuttle vectors.

Once the shuttle vectors were confirmed, they were linearized with PmeI (NEB, Ipswich, MA) and recombined into the pAdEasy backbone [47]. Two control vectors, containing wild type pIX were used for this work, AdLuc1 and AdCMVLuc (kindly provided by Dr Igor Dmitriev, UAB), both containing the wild type pIX, but with slightly differing backbone. AdLuc1 was used only in ELISA experiments. For gene transfer experiments the control vector AdCMVLuc made from the identical parental shuttle plasmid (pSI) was used to provide a better comparison. Viral genomes and rescued viruses were termed AdLucIXLinker-ligand.

Rescue and propagation of viruses was carried out on HEK 293 cells (success/failure summarized in Table 1A), and viruses were purified from infected cells by three freezethaw cycles followed by two successive bandings on cesium chloride ( $\mathrm{CsCl}$, Sigma, St. Louis, MO) gradients. Particle units $(\mathrm{pu})$ of viruses were determined with standard OD260 methods. Infectious virus titer was determined by focus forming unit (ffu) assay. Monolayers of HEK 293 cells were infected with serial dilutions of the vector. Following a suitable incubation period, the infected cells were probed with an adenovirus antibody and than with a secondary antibody conjugated with Fluorescein-Isothiocyanate (FITC). Fluorescent foci are viewed under a UV microscope and enumerated. Specific viral concentrations and titers are listed in Table $\mathbf{1 B}$.

\section{Analysis of pIX-Modified Protein Capsid Incorporation by Western Blot}

Virus ( $5 \times 10^{9} \mathrm{pu}$ per sample) was denatured by boiling in Laemmli loading buffer (Bio-Rad, Hercules, CA). The viral capsid proteins were separated by a 4-20\% gradient polyacrylamide gel (Bio-Rad) and the electrophorectically resolved viral capsomers were transferred to polyvinylidenedifluoride membrane (Bio-Rad) and probed with anti-FLAG monoclonal antibody (Sigma) as all pIX fusions contain a FLAG motif as previously described [29]. The blots were developed with the WesternBreeze Immunodetection system (Invitrogen, Carlsbad, CA) according to manufacturer's protocol. Additionally during propagation cell lysates from cells infected with vectors were also tested. In this case $10 \mu \mathrm{l}$ of 293 cell lysates from the rounds of propagation were instead denatured by boiling in Laemmli loading buffer and analyzed on western blot using the anti-FLAG antibody.

\section{Assessment of ABD Adenovirus Vector Binding to Albumin}

Human, murine, bovine or canine albumin (all obtained from Sigma) prepared at $5 \mu \mathrm{g} / \mathrm{ml}$ were adsorbed to 96 -well Nunc Immuno-plates plates (Fisher Scientific, Pittsburgh, PA) overnight in $100 \mu \mathrm{l}$ binding buffer $(50 \mathrm{mM}$ carbonate buffer $(\mathrm{Ph} 8.6))$ at $4^{\circ} \mathrm{C}$. An additional plate was treated with binding buffer alone (data not shown, as identical to bovine). The following day, wells were blocked with $0.5 \%$ casein prepared in $1 \mathrm{xTBS} / 0.05 \%$ Tween and then viruses were added at the above pu/well (highest $30 \times 10^{9} \mathrm{pu} /$ well) diluted in blocking buffer. In between all steps wells were washed with 1 XTBS $/ 0.05 \%$ Tween. Wells were probed for bound adenovirus particles with anti-Ad2 polyclonal antibody (NIAID) for $1 \mathrm{hr}$ at RT and then with the secondary HRP antibody being goat anti-rabbit immunoglobulin antibody (Dako, Carpinteria, CA) for 1 hour at RT. The color was developed with the Sigma FAST o-phenylenediamine dihydrochloride tablet kit (Sigma) as recommended by the manufacturer and then fixed with 1N HCL. The color intensity was measured at $450 \mathrm{~nm}$ with an EL800 plate reader (BioTek Instruments, Winooski, VT). It should be noted that the presence of the various albumins in the ELISA plates were probed in separate wells with specific anti-albumin antibodies (data not shown, antibodies from Bethyl Laboratories, Montgomery, TX) and it was confirmed that the albumins absorbed to the plates. 
Table 1A. Analysis of pIX-Modified Vector Rescue, Propagation and Incorporation of pIX-Protein Fusions into the Ad Vector Capsid

\begin{tabular}{|c|c|c|c|c|c|c|}
\hline \multirow{2}{*}{$\begin{array}{c}\text { pIX Configuration } \\
\text { Protein }\end{array}$} & \multicolumn{2}{|l|}{ pIXFlag } & \multicolumn{2}{|l|}{ pIX45A } & \multicolumn{2}{|l|}{ pIX75A } \\
\hline & Rescue \& Propagation & WB & Rescue \& Propagation & WB & Rescue \& Propagation & WB \\
\hline Stop Codon & Yes & Yes & Yes & Yes & Yes & Yes \\
\hline \multicolumn{7}{|l|}{ Direct Concept Vectors } \\
\hline $\mathrm{A} 1 \mathrm{~A}\left(\mathrm{P} 1-\mathrm{P} 1{ }^{\prime}\right)$ & Yes & Yes & Yes & Yes & ND & ND \\
\hline sr39TK & Yes & Yes & ND & ND & ND & ND \\
\hline hALB & Yes/No & $\mathrm{ND}^{*}$ & ND & ND & Yes/No & ND \\
\hline \multicolumn{7}{|c|}{ Indirect Concept Vectors } \\
\hline
\end{tabular}

WB Western Blot detection of pIX-fusion in capsid of $\mathrm{CsCl}$ purified vectors. ND Not Done.

hALB Yes//No - Virus could be rescued but was not successfully propagated.

ND* Not Done on $\mathrm{CsCl}$ purified vector.

Table 1B. Viral Titers and pu/ffu Ratio Attained for Successfully Propagated pIX-Modified Vectors

\begin{tabular}{|c|c|c|c|c|}
\hline & Virus Name & Particle Concentration (pu/ml) & Titer (ffu/ml) & Ratio (pu/ffu) \\
\hline \hline 1 & AdCMVLuc & $1.2 \times 10^{12}$ & $2.0 \times 10^{10}$ & 30 \\
\hline 2 & AdLuc.IXFlag-stop & $1.7 \times 10^{12}$ & $4.4 \times 10^{10}$ & 38 \\
\hline 3 & AdLuc.IXFlag-ABD & $1.5 \times 10^{12}$ & $4.0 \times 10^{10}$ & 43 \\
\hline 4 & AdLuc.IX45A-stop & $1.7 \times 10^{12}$ & $2.6 \times 10^{10}$ & 46 \\
\hline 5 & AdLuc.IX45A-ABD & $1.2 \times 10^{12}$ & $7.1 \times 10^{10}$ & 17 \\
\hline 6 & AdLuc.IX75A-stop & $1.2 \times 10^{12}$ & $4.7 \times 10^{9}$ & 68 \\
\hline 7 & AdLuc.IX75A-ABD & $3.2 \times 10^{11}$ & $2.7 \times 10^{10}$ & 44 \\
\hline 8 & AdLuc.IXFlag-sr39TK & $1.2 \times 10^{12}$ & $4.7 \times 10^{9}$ & 117 \\
\hline 9 & AdLuc.IXFlag-A1A & $5.5 \times 10^{11}$ & $7.3 \times 10^{9}$ & 192 \\
\hline 10 & AdLuc.IXFlag-A1A(P1-P1') & $1.4 \times 10^{12}$ & $2.1 \times 10^{9}$ & $1.4 \times 10^{10}$ \\
\hline 11 & AdLuc.IX45A-A1A & $4.6 \times 10^{11}$ & $1.8 \times 10^{12}$ & 129 \\
\hline 12 & AdLuc.IX45A-A1A(P1-P1') & & & \\
\hline
\end{tabular}

\section{Adenoviral Infection of A549 Cells to Assess pIX-Protein Shielding Properties}

A549 cells were seeded into 96 well plates at $1 \times 10^{4}$ cells/well 24 hours before infection with adenovirus vectors at an MOI of $100 \mathrm{pu} / \mathrm{cell}$. An MOI of $100 \mathrm{pu} / \mathrm{cell}$ is a standard quantity to use with A549 cells as demonstrated in numerous studies. In studies with A1A or sr39TK containing vectors (and appropriate controls), vectors were incubated for 30 minutes at $37^{\circ} \mathrm{C}$ with a 1:2500 dilution of anti-Ad5 antibody (Abcam, Cambridge, MA) or were incubated for 30 minutes at $37^{\circ} \mathrm{C}$ with 1:2500 dilution of polyclonal hexon antibody (GeneTex, Inc., San Antonio, TX) pre-infection. Cells were then incubated with virus for 2 hours at $37^{\circ} \mathrm{C}$, washed with PBS and refed with normal media. After 24 hours cells were lyzed with Luciferase Reporter Lysis Buffer (Promega, Madison, WI) and assayed for luciferase activity, measured as relative light units (RLU) according to the manufacturer protocol (Promega Luciferase Kit). The 1:2500 dilution of anti-Ad antibody and anti-hexon antibody was determined by mixing AdCMVLuc with a serial dilution of the antibodies prior to infection of cells, and determining a dilution that resulted in a $2 \log$ reduction in luciferase activity when compared to AdCMVLuc with no antibody (data not shown). Viruses were also assessed after pre-mixing with naïve or immunized mouse serum at 1, 1025,50 and $90 \%$ serum concentration. Infection of cells and luciferase activity was assessed as previously described. Naïve serum was obtained from C57BL/6 mice (The Jackson Laboratory, Bar Harbor, ME) that were not exposed to wt Ad5 vector while 
immunized serum was obtained from C57BL/6 mice 14 days following tail vein injection $10^{9} \mathrm{pu} /$ mouse of wt Ad5 vector (HAdV-5, ATCC, formerly known as Adwt300 [48].

Two sets of experiment were carried out with the ABD containing vectors. In the first experiment, AdLucIX45A$\mathrm{ABD}$ and control vector, AdLucIX45A-Stop, prior to antibody incubation, were pre-mixed with varying quantities of hALB (Sigma) for 30 minutes at $37^{\circ} \mathrm{C}$. All viruses, including AdCMVLuc were then incubated without or with a 1:2500 dilution of anti-Ad5 antibody (Abcam) for 30 minutes at $37^{\circ} \mathrm{C}$. Cells were then incubated with virus for 2 hours at $37^{\circ} \mathrm{C}$, washed with PBS and refed with normal media. A further 24 hours later, cells were analyzed for luciferase activity using standard methods. In the second experiment preincubation the vectors were incubated for 30 minutes at $37^{\circ} \mathrm{C}$ in serum free media, with $90 \%$ naïve murine serum or $90 \%$ serum from mice immunized with Ad5. Naïve serum was obtained as above, but this time the immunized serum was obtained from C57BL/6 mice 28 days following tail vein injection $10^{9} \mathrm{pu} /$ mouse of wt Ad5 vector. Following this initial incubation, the pre-mixes were then either incubated without antibody or with a 1:2500 dilution of anti-Ad5 antibody (Abcam) for a further 30 minutes at $37^{\circ} \mathrm{C}$. Cells were then incubated with virus for 2 hours at $37^{\circ} \mathrm{C}$, washed with PBS and refed with normal media. A further 24 hours later, cells were analyzed for luciferase activity using standard methods. All gene transfer experiments were carried out once but with triplicates within the actual experiment. The experiments are represented at relative light units (RLU) for luciferase activity. The data was also normalized but the patterns per results remained identical (data not shown).

\section{RESULTS}

\section{Concept 1: Assessment of Shielding Capacity of pIX Modified Ad Vectors Containing Shield Protein Directly Fused to pIX}

The preferred method for genetic shielding would be to directly incorporate hALB into Ad5 vectors through genetic fusion with pIX (Fig. 1) We also used A1A and A1A (P1-P1') along with sr39TK to test as shielding molecules in this concept. The molecules A1A and the enzymatic deficient $\mathrm{A} 1 \mathrm{~A}(\mathrm{P} 1-\mathrm{P} 1$ ') were included as alternate self molecules while sr39TK was included because it is a proven pIX modification [31]. All the Ad vectors were generated using the pAdEasy system [47] and pIX_modified Ad vectors containing sr39TK and A1A molecules were rescued and propagated on 293 cells (as indicated in Table 1A). Successful capsid incorporation of modified pIX proteins was indicated through western blot analysis (sr39TK is shown in Fig. (2), panel A). Following a similar propagation strategy to that used with control Ad vector, AdCMVLuc, these vectors could be propagated to titers between $5 \mathrm{x} 10^{11} \mathrm{pu} / \mathrm{ml}$ and $2 \times 10^{12} \mathrm{pu} / \mathrm{ml}$. While the pu/ffu ratio of pIX-sr39TK was at 44 those of the A1A related vectors were higher falling between 117 and 219 (Table 1B). The current batch of AdCMVLuc had a pu/ffu ratio of 60 . However difficulties arose with generating viruses with hALB fused directly to pIX.

Initially only the AdLucIXFlag-hALB genome was prepared as proteins larger than hALB, TK-luciferase [36] and TK-eGFP[35], have been successfully fused onto the shortest pIX-flag variant resulting in capsid incorporation. Plaques that arose on transfected 293 cells indicated that virus was
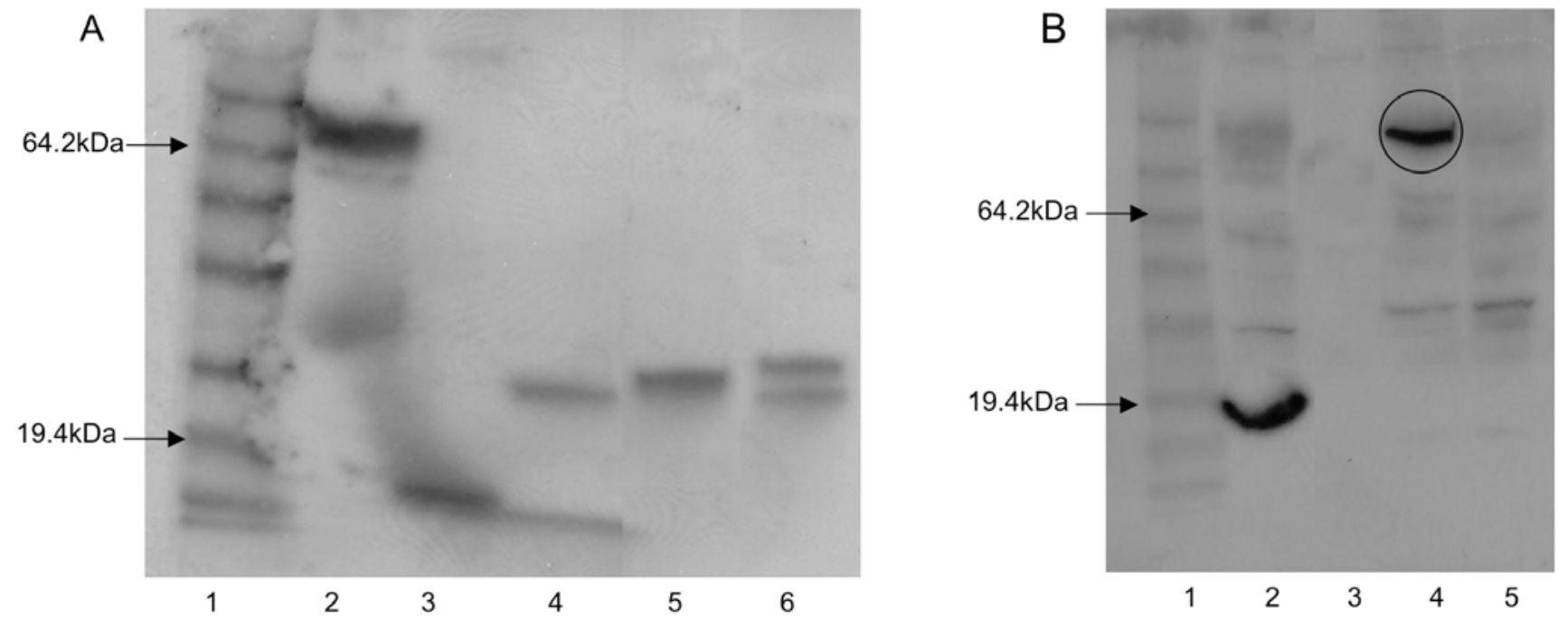

Fig. (2). Analysis of capsid incorporation of pIX-molecule fusions (panel A) and production of pIX-molecule fusions in cell lysates (panel B). $\mathrm{CsCl}$ purified virions of pIX-modified Ad vectors were analyzed for pIX-molecule capsid incorporation through western blot analysis (panel A). Ad vectors were loaded at $5 \times 10^{9}$ pu/lane after boiling and samples probed with anti-FLAG antibody. Lane order is as follows: (1) Marker, (2) AdLucIX-sr39TK (57kDa), (3) AdLucIXFlag-Stop (16kDa), (4) AdLucIXFlag-ABD (21kDa), (5) AdLucIX45A-ABD (25kDa) and (6) AdLucIX75A-ABD (28kDa). Cell lysates from 293 cells infected with AdLucIXFlag-hALB were analyzed for production of the pIX fusion during rescue and two rounds of propagation of vector (panel B). Lysate from cells infected with AdLucIXFlag-ABD was used as control for anti-FLAG antibody. Lane order is as follows: (1) Marker, (2) AdLucIXFlag-ABD, (3) AdLucIXFlag-hALB at rescue (35mm dish), (4) AdLucIXFlag-hALB at round one propagation and (5) AdLucIXFlag-hALB at round two of propagation. The presence of pIXhALB $(83 \mathrm{kDa})$ in the cell lysate is circled and only seen at round one propagation. 
rescued. However propagation of the virus to $\mathrm{CsCl}$ purification stage was unsuccessful due to ever increasing length of time, outside of the normal 2-3 days, to see cytopathic effect (CPE) on the infected cells. The gene fusion could be detected by PCR analysis of the cell lysates from infected cells (data not shown) but the protein was only detected in the second round of amplification (where CPE occurred at 4 days) by western blot (Fig. 2, panel B). This also suggested that the fusion protein probably is not aggregating due to a lack of a specific band at higher molecular weight. A propagation strategy of harvesting cells every four days after virus infection was also attempted but no CPE was seen and resulted in empty capsids during $\mathrm{CsCl}$ purification. Therefore these hALB vectors were not pursued in tests of gene transfer experiments.

To determine if the capsid incorporated pIXFlag-A1A and sr39TK fusions could provide a shielding effect in vitro gene transfer analysis was used as the starting point. Briefly viruses were pre-mixed with neutralizing antibodies, or just in serum free media, and then luciferase activity was used to determine the level of infection of cells with vectors. Adenovirus infection properties of several pIX-modified vectors, including control vectors (AdLucIXFlag-stop and AdLucIX45A-stop), in the absence and presence of anti-Ad5 (1:2500 dilution) (Fig. 3, panel A), were analyzed to determine if any of the modifications provided a shielding effect. The results demonstrated that the modified pIX region did not affect general infection properties of the vectors when compared to AdCMVLuc. However the results also demonstrated that none of the proteins fused to pIX provided a shielding effect, as the antibody effectively reduced luciferase activity for all vectors tested. We also tested serum from naïve and immunized C57BL/6 mice (serum taken 14 days after immunization with a wild type vector) and com-
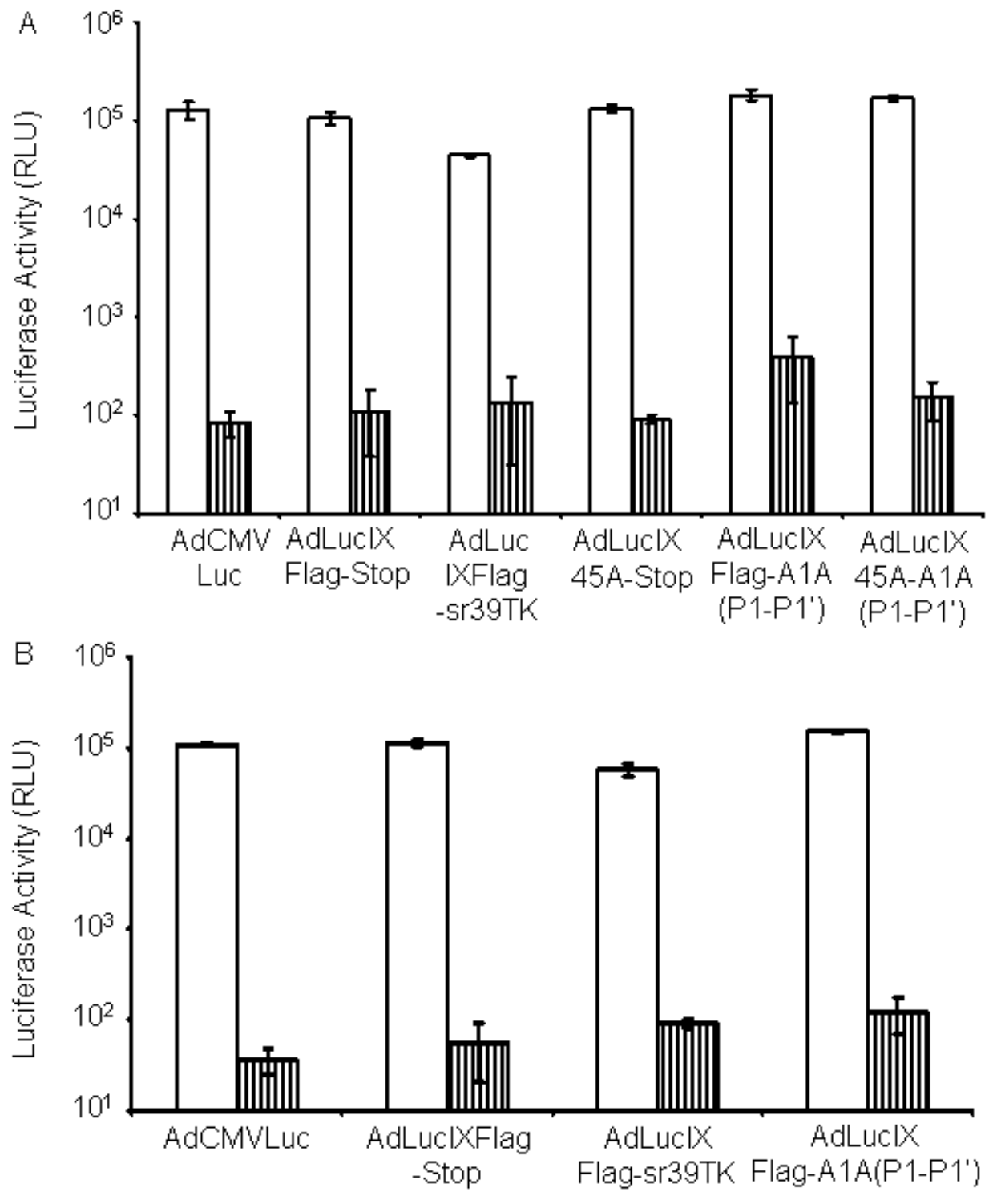

Fig. (3). Effect of neutralizing antibody on gene transfer of Ad5 vectors with direct incorporation of shielding proteins was assessed 24 hours following infection of A549 cells. Luciferase activity expressed as relative light units (RLU) is compared between vectors that were incubated with either a 1:2500 dilution of anti-Ad5 antibody (panel A) or a 1:2500 dilution of polyclonal hexon antibody (panel B) (striped columns) and vectors without antibody pre-incubation (white columns, both panels). All of the measurements are shown as mean RLU ( $=3$, +/- standard deviation). 
pared $1 \%, 10 \%, 25 \%, 50 \%$ and $90 \%$ serum first on AdCMVLuc, and found that there was 0.5 to $1 \log$ reduction in luciferase activity at $50 \%$ and $90 \%$ serum respectively (data not shown). This result may also suggest that there is less anti-Ad5 antibody present in immunized mouse serum than in the polyclonal antibody preparation but apart from this crude analysis, neutralizing titer of the serum was not compared to the polyclonal antibody preparation. When we compared AdCMVLuc, AdLucIXFlag-sr39TK and AdLucIX45-A1A at the 50\% serum concentration, a similar reduction in luciferase activity was seen with all three viruses indicating that no shielding effect was seen (data not shown).

To assess whether any of the proteins provided some shielding of immunogenic epitopes a polyclonal hexon antibody was used. Hexon proteins form the largest surface on the capsid with the most dominant epitopes and consequently shielding against hexon neutralizing antibodies would be very important [49]. While penton and fiber also have immunogenic epitopes, we used the logic that inability of the pIX attached molecules to shield against hexon, probably would not allow shielding against penton and fiber neutralizing antibodies. This is due to the spatial arrangement of proteins in the capsid, especially fiber which extends away from the capsid surface and therefore decided to only test a hexon antibody. The adenovirus infection properties of AdLucIXFlag-Stop, AdLucIXFlag-sr39TK and AdLucIXFlag-A1A (P1-P1') pre-mixed, with or without anti-hexon antibody (used at the 1:2500 dilution), were then analyzed (Fig. 3, panel B). The results clearly demonstrated that the poly- clonal hexon antibody was able to neutralize adenovirus infection regardless of which protein was fused to pIX.

\section{Concept 2: Assessment of Indirect Shielding Capacity of pIX Modified Ad Vectors Containing a Binding Motif Conjugated to Shield Protein}

As with the hALB, A1A, A1A(P1-P1') and sr39TK vectors for strategy one, we generated $\mathrm{ABD}$ vectors with pAdEasy as indicated in Table 1. All three ABD vectors and their corresponding control vectors (pIX-linker-stop) could be rescued and propagated on 293 cells, to attaining titers above $1-2 \times 10^{12} \mathrm{pu} / \mathrm{ml}$ under similar propagation strategies to AdCMVLuc. Only AdLucIX75A-ABD did not grow to a high titer $\left(<5 \times 10^{11} \mathrm{pu} / \mathrm{ml}\right)$. However all these vectors had $\mathrm{pu} / \mathrm{ffu}$ ratios within the 17 to 68 range (see Table 1B). The pIX fusions were detected through western blot analysis (Fig. 2, panel A). One interesting facet for AdLucIX75A$\mathrm{ABD}$ was that two pIX bands were detected on western blot suggesting that this construct is not completely stable and that perhaps the ABD portion on some of the pIX-fusions is being degraded. This data corroborates another report that the $75 \AA$ linker is not necessarily the best linker for pIX attached protein constructs [50].

Prior to gene transfer experiments, the functionality of $\mathrm{ABD}$ in the pIX capsid incorporated context was tested through ELISA methodology (Fig. 4). Albumins from various sources, human (Fig. 4, panel A), murine (Fig. 4, panel B), bovine (Fig. 4, panel C) and canine (Fig. 4, panel D) were adsorbed to ELISA plates and then the appropriate

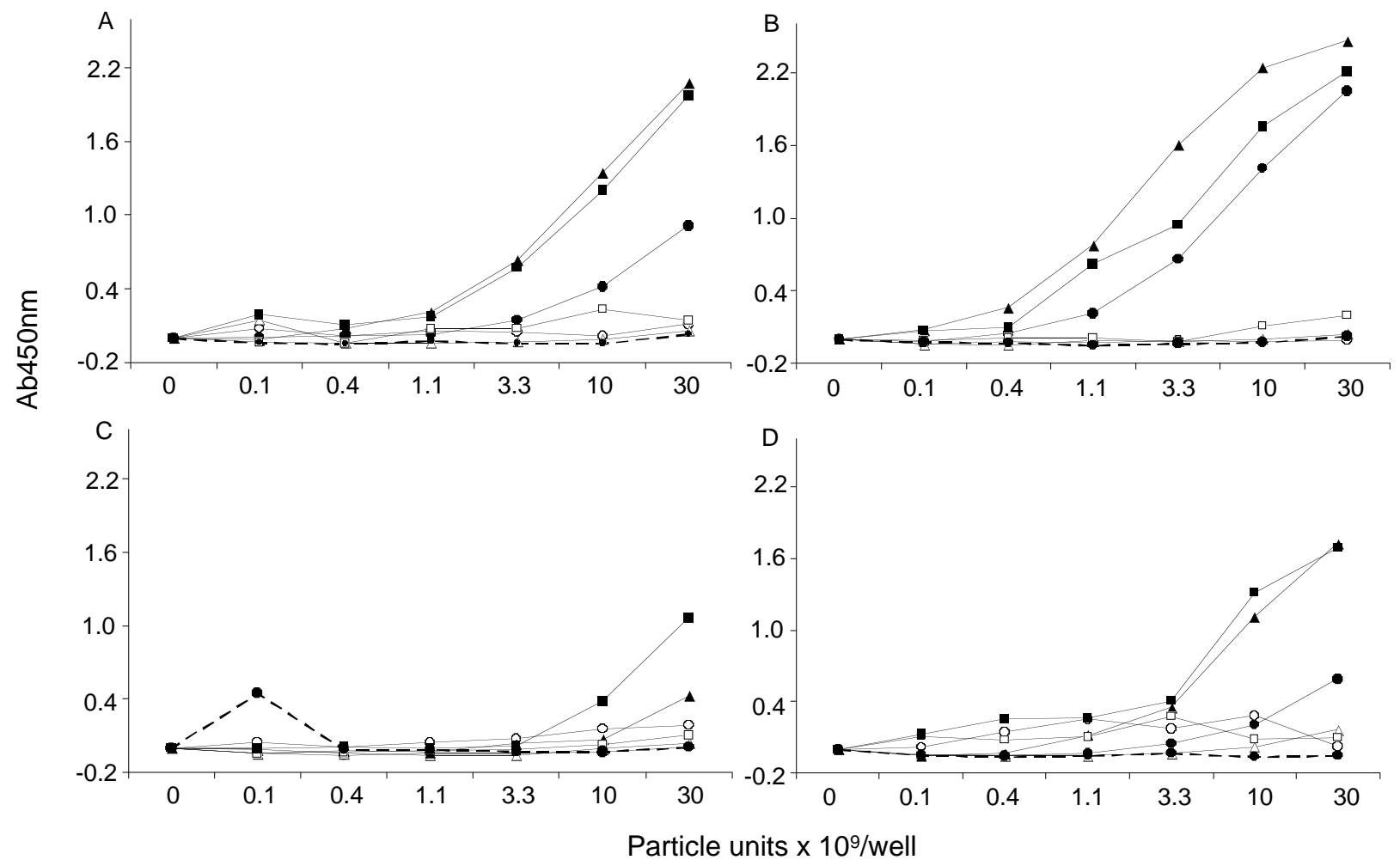

Fig. (4). Analysis of Ad5 capsid incorporated pIX-ABD interaction with human (panel A), murine (panel B), bovine (panel C) or canine (panel D) albumin through ELISA methodology with increased binding indicated by increase in absorbance reading. The viruses are represented as follows: AdLucIXFlag-ABD (solid circle), AdLucIXFlag-Stop (open circle), AdLucIX45A-ABD (solid triangle), AdLucIX45AStop (open triangle), AdLucIX75A-ABD (solid square), AdLucIX75A-Stop (open square), AdLuc1 (dashed line). 
$\mathrm{ABD}$ and control viruses were added to the wells. The $\mathrm{ABD}$ viruses had most affinity with hALB (Fig. 4, panel A) and murine albumin (mALB) (Fig. 4, panel B) by virtue of the higher OD readings obtained when compared to control viruses at concentrations greater than $3.3 \times 10^{9} \mathrm{pu} /$ well and 1.1 x $10^{9} \mathrm{pu} /$ well respectively. There was less affinity with canine albumin (cALB) (Fig. 4, panel D), and essentially no affinity with bovine (Fig. 4, panel C) under these conditions for the ABD viruses. No affinity with plastic was detected for these viruses (data not shown) indicating that the results seen with hALB and mALB were specific for the presence of these albumins adsorbed to the plates. In addition all plates were tested with albumin control antibodies to confirm adsorption to plates (data not shown). AdLucIX45A-ABD (solid triangles) performed the best with mALB (Fig. 4, panel B) and was similar with AdLucIX75A-ABD (solid squares) on both hALB (Fig. 4, panel A) and cALB (Fig. 4, panel D). However, due to the hinted instability of AdLucIX75A-ABD it was decided that AdLucIX45A-ABD would be the ABD vector of choice to analyze in gene transfer experiments.

The ability of AdLucIX45A-ABD and control vector AdLucIX45A-Stop to infect cells was then analyzed following pre-mixing with a serial dilution of hALB, and after the initial incubation period, mixed with or without anti-Ad5 (used at the 1:2500 dilution) (Fig. 5, panel A). AdCMVLuc, without hALB, but +/- antibody was used as a benchmark for
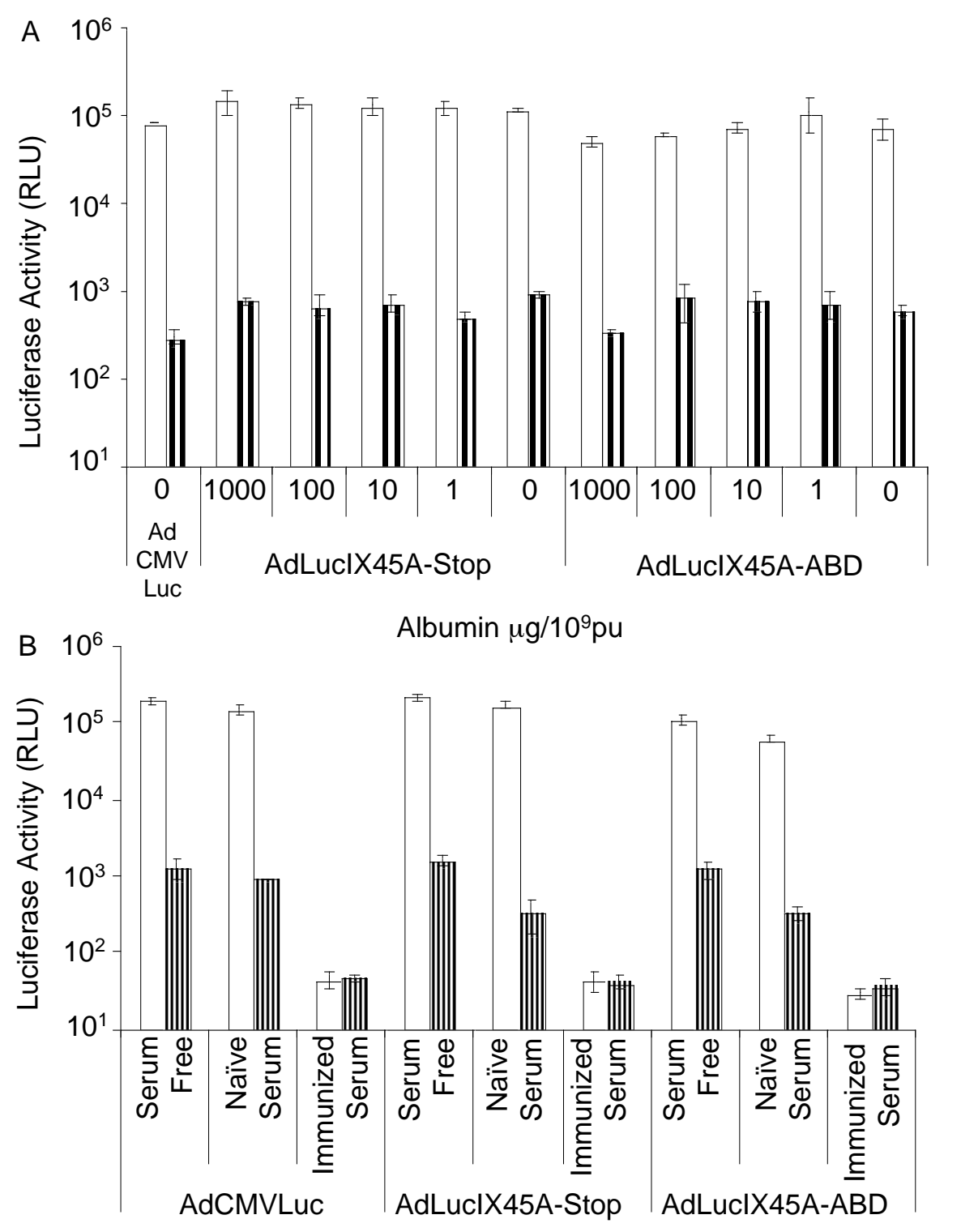

Fig. (5). Effect of anti-Ad5 antibody neutralizing capacity on gene transfer of adenovirus vectors with indirect incorporation of hALB conjugation (panel A) or mALB (panel B) was assessed 24 hours following infection of A549 cells. In panel A luciferase activity expressed as RLU is compared between vectors that were pre-mixed with varying quantities of hALB (Sigma) and then incubated with a 1:2500 dilution of anti-Ad5 antibody (striped columns) or no antibody incubation (white columns) (panel A). In panel B luciferase activity is compared between vectors that were pre-mixed with in serum free media, with $90 \%$ naïve murine serum or $90 \%$ serum from mice immunized with Ad5 and then incubated with a 1:2500 dilution of anti-Ad5 antibody (striped columns) or no antibody incubation (white columns) (panel B). In panel A, AdCMVLuc was not pre-incubated with hALB, but was pre-incubated +/- anti-Ad5 antibody. All of the measurements are shown as mean RLU ( $\mathrm{n}=3,+/$ - standard deviation). 
determining success or failure of the experiment. As expected the control AdLucIX45A-Stop was neutralized under all conditions, in the same manner as AdCMVLuc. However, AdLucIX-45A-ABD was also neutralized under all conditions. This result indicated two possibilities. The first possibility is that the pre-incubation with hALB did not form an Ad-albumin complex which conflicts with the ELISA data where it was clear that AdLucIX45A-ABD could adhere to hALB coated plates. Secondly, if the virus was coated with albumin then the albumin was not providing a shield against neutralizing antibodies.

To further assess whether AdLucIX45A-ABD could bind albumin and attain a shielding affect, the virus was used in an experiment without prior coating to hALB. Instead the vector was mixed with serum from naïve mice (C57BL/6 murine model) and from mice that had been pre-immunized with wild type Ad5 vector. It was hypothesized that in naïve serum the vector would conjugate with mALB in the serum (based on the positive ELISA data, Fig. 4) and the mALB would prevent neutralization by antibodies against Ad5 once the polyclonal antibody was added to the mix. It was a little less clear as to what would happen with immunized serum, as this already contains neutralizing antibodies, and there would potentially be competition between the albumin and antibodies in this serum. Due to the results from the control vector with $90 \%$ serum in section one of the results where a $\log$ decrease was seen in gene transfer it was decided to obtain serum from mice at a 28 day time period following wild type adenovirus administration. The $90 \%$ concentration of serum was again used in these experiments. Following preincubation of AdLucIX45A-ABD (and control vectors) in serum free media with naïve and immunized murine serum, the polyclonal antibody against Ad5 was added to the mix (Fig. 5, panel B). In serum free control conditions AdLucIX45A-ABD was neutralized as expected, while in immunized serum, it was clear that murine anti-Ad5 antibodies are able to neutralize Ad vector infection. Furthermore the reduction of luciferase activity from AdLucIX45A-ABD in naïve serum conditions with anti-Ad5 illustrates that either insufficient mALB was bound to the vector or albumin conjugated to the capsid is not capable of preventing neutralization. The conclusion from this part of the work was that this particular binding motif, ABD and albumin (human or murine) were not capable of providing a shielding effect.

\section{DISCUSSION AND CONCLUSION}

The data demonstrated several issues with pIX fusions and the potential of genetic shielding. This is the first study to show that not all molecules fused to pIX can be successfully incorporated into the Ad vector capsid. In this case hALB was deemed incompatible with direct capsid incorporation and it could be speculated that the $83 \mathrm{kDa}$ pIXFlaghALB fusion appeared to have inhibitory actions on the formation of virions. While capsid assembly is supposedly not affected by the absence of pIX [51], it is thought to play a role in packaging full length genomes [52]. Furthermore, while the product of the adenovirus intermediate gene IX is a transcriptional activator it is not absolutely required [53]. Albumin is not known to bind DNA and therefore this should not be a cause for the problems seen here. Although pIXFlag-hALB fusions could be detected in an early round of propagation, perhaps the disulphide complexity of the albumin and/or some other factor makes this protein unstable and ultimately unsuitable for capsid incorporation leading to inefficient packaging of genomes. Based on the western blot results we concluded that the pIXFlag-hALB was not aggregating. It would seem virions with full length genomes are attained in early rounds of propagation, but empty capsids become more predominant as the number of rounds of propagation increase. The ABD ELISA data also indicates that there may be spatial factors playing a role with albumin, in that longer linkers were required to see good conjugation to albumin, thus suggesting the natural globular configuration of albumin may prevent it from fitting into the pIX cavity. Currently the classical configuration of the Ad capsid and position of pIX is being questioned [54] and therefore spatial elements of the capsid may play a bigger role in determining suitable large pIX-protein fusions to develop as pIX-modified vectors. Despite the failure with albumin a smaller self-protein alpha-1-antitrypsin was successfully fused to the pIX protein and virus was generated with this fusion capsid incorporated. This is the first report of a large protein, not functionally limited to imaging applications, fused to the pIX and broadens the scope of proteins that can be fused to pIX.

The data obtained from the in vitro shielding experiments showed that (a) direct incorporation of proteins of $\sim 60 \mathrm{kDa}$ (A1A) or less cannot provide sufficient coverage of the highly immunogenic hexon regions of the adenovirus capsid and (b) indirect incorporation of albumin to the adenovirus capsid through the ABD protein did not provide shielding to the vector. This is based on the significant reduction in adenovirus infection of the pIX-modified vectors in the presence of anti-Ad5 antibody (for all viruses) and in the presence of anti-hexon antibody (for sr39TK and A1A(P1-P1'). Certainly the hexon data indicates our molecules are not large enough to cover the immunogenic epitopes of hexon. The data for sr39TK is contradictory to our initial report that a pIXFlag-sr39TK virus can provide a shielding effect [28]. The first report was based on a static ELISA method and the results illustrated here are based on a 3 dimensional experiment with serum mixed with virus prior to gene transfer. Therefore this study indicates the need to use suitable methodologies when examining concepts.

Despite the problems with pIXFlag-hALB, a larger protein, a TK-luciferase fusion (over $90 \mathrm{kDa}$ ) has been successfully incorporated into pIX-modified virions with just the standard FLAG motif [36] indicating larger self-proteins could be explored as shielding agents. The absolute size limitations for pIX-fusions have yet to be determined but other considerations such as disulphide complexity, and post-translational modifications will need to be accounted for when looking for a suitable self-protein. Even if a suitably large self-protein was determined and could show hexon coverage, then modifications to the fiber may also be required to block neutralizing antibody sites on the fiber due to the extension away from the capsid of this protein. Two recent reports have indicated that fiber modifications may help Ad vectors escape the immune response [55, 56], and therefore this would certainly be something that requires further investigation in combination with finding suitable alternate shielding molecules. 
ABBREVIATIONS

$\begin{array}{ll}\mathrm{A} 1 \mathrm{~A} & =\text { Alpha-1-antitrypsin } \\ \mathrm{ABD} & =\text { Albumin binding domain } 3 \\ \mathrm{Ad} & =\text { Adenovirus } \\ \mathrm{cALB} & =\text { Canine albumin } \\ \mathrm{CPE} & =\text { Cytopathic effect } \\ \mathrm{CRAd} & =\text { Conditionally replicative Ad vectors } \\ \mathrm{CsCl} & =\text { Cesium chloride } \\ \mathrm{ds} \mathrm{cDNA} & =\text { Double stranded cDNA } \\ \mathrm{ffu} & =\text { Focus forming unit } \\ \mathrm{hALB} & =\text { Human albumin } \\ \mathrm{mALB} & =\text { Murine albumin } \\ \text { pu } & =\text { Particle unit } \\ \text { rHSA } & =\text { Recombinant human serum albumin } \\ \mathrm{RLU} & =\text { Relative light units }\end{array}$

\section{ACKNOWLEDGEMENTS}

We would like to thank Professor David A Lomas (University of Cambridge, UK) for his advice with regards to the alpha-1-antitrypsin mutation generated in this paper. This work was funded by NIH SBIR grants 1R43AI072894-01A1 and 1R43CA123768-01A1.

\section{CONFLICT OF INTEREST}

David T. Curiel is an equity holder in VectorLogics, Inc.

\section{REFERENCES}

[1] Vogels R, Zuijdgeest D, van Rijnsoever R, et al. Replicationdeficient human adenovirus type 35 vectors for gene transfer and vaccination: efficient human cell infection and bypass of preexisting adenovirus immunity. J Virol 2003; 77: 8263-71.

[2] Nwanegbo E, Vardas E, Gao W, et al. Prevalence of neutralizing antibodies to adenoviral serotypes 5 and 35 in the adult populations of The Gambia, South Africa, and the United States. Clin Diagn Lab Immunol 2004; 11: 351-7.

[3] Chen P, Kovesdi I, Bruder JT. Effective repeat administration with adenovirus vectors to the muscle. Gene Ther 2000; 7: 587-95.

[4] Davis JJ, Fang B. Oncolytic virotherapy for cancer treatment: challenges and solutions. J Gene Med 2005; 7: 1380-9.

[5] Seshidhar Reddy P, Ganesh S, Limbach MP, et al. Development of adenovirus serotype 35 as a gene transfer vector. Virology 2003; 311: 384-93.

[6] Gao W, Robbins PD, Gambotto A. Human adenovirus type 35: nucleotide sequence and vector development. Gene Ther 2003; 10: 1941-9.

[7] Holterman L, Vogels R, van der Vlugt R, et al. Novel replicationincompetent vector derived from adenovirus type 11 (Ad11) for vaccination and gene therapy: low seroprevalence and non-crossreactivity with Ad5. J Virol 2004; 78: 13207-15.

[8] Capone S, Meola A, Ercole BB, et al. A novel adenovirus type 6 (Ad6)-based hepatitis $\mathrm{C}$ virus vector that overcomes preexisting anti-ad5 immunity and induces potent and broad cellular immune responses in rhesus macaques. J Virol 2006; 80: 1688-99.

[9] Ruzsics Z, Wagner M, Osterlehner A, Cook J, Koszinowski U, Burgert HG. Transposon-assisted cloning and traceless mutagenesis of adenoviruses: development of a novel vector based on species D. J Virol 2006; 80: 8100-13.

[10] Thirion C, Lochmuller H, Ruzsics Z, et al. Adenovirus vectors based on human adenovirus type 19a have high potential for human muscle-directed gene therapy. Hum Gene Ther 2006; 17: 193-205.
[11] Lemiale F, Haddada H, Nabel GJ, Brough DE, King CR, Gall JG. Novel adenovirus vaccine vectors based on the enteric-tropic serotype 41. Vaccine 2007; 25: 2074-84

[12] Bangari DS, Mittal SK. Development of nonhuman adenoviruses as vaccine vectors. Vaccine 2006; 24: 849-62.

[13] Le LP, Li J, Ternovoi VV, Siegal GP, Curiel DT. Fluorescently tagged canine adenovirus via modification with protein IX enhanced green fluorescent protein. J Gen Virol 2005; 86: 3201-8.

[14] Roy S, Zhi Y, Kobinger GP, et al. Generation of an adenoviral vaccine vector based on simian adenovirus 21. J Gen Virol 2006; 87: 2477-85.

[15] Roy S, Kobinger GP, Lin J, et al. Partial protection against H5N1 influenza in mice with a single dose of a chimpanzee adenovirus vector expressing nucleoprotein. Vaccine 2007; 25: 6845-51.

[16] Hashimoto M, Boyer JL, Hackett NR, Wilson JM, Crystal RG. Induction of protective immunity to anthrax lethal toxin with a nonhuman primate adenovirus-based vaccine in the presence of preexisting anti-human adenovirus immunity. Infect Immun 2005; 73: 6885-91.

[17] Zhang Y, Bergelson JM. Adenovirus receptors. J Virol 2005; 79: 12125-31.

[18] Iacobelli-Martinez M, Nepomuceno RR, Connolly J, Nemerow GR. CD46-utilizing adenoviruses inhibit C/EBPbeta-dependent expression of proinflammatory cytokines. J Virol 2005; 79: 11259-68.

[19] Eto Y, Gao JQ, Sekiguchi F, et al. Neutralizing antibody evasion ability of adenovirus vector induced by the bioconjugation of methoxypolyethylene glycol succinimidyl propionate (MPEG-SPA). Biol Pharm Bull 2004; 27: 936-8.

[20] Chillon M, Lee JH, Fasbender A, Welsh MJ. Adenovirus complexed with polyethylene glycol and cationic lipid is shielded from neutralizing antibodies in vitro. Gene Ther 1998; 5: 995-1002.

[21] Mok H, Palmer DJ, Ng P, Barry MA. Evaluation of polyethylene glycol modification of first-generation and helper-dependent adenoviral vectors to reduce innate immune responses. Mol Ther 2005; 11: 66-79.

[22] Romanczuk H, Galer CE, Zabner J, Barsomian G, Wadsworth SC O'Riordan CR. Modification of an adenoviral vector with biologically selected peptides: a novel strategy for gene delivery to cells of choice. Hum Gene Ther 1999; 10: 2615-26.

[23] O'Riordan CR, Lachapelle A, Delgado C, et al. PEGylation of adenovirus with retention of infectivity and protection from neutralizing antibody in vitro and in vivo. Hum Gene Ther 1999; 10: 1349-58.

[24] Croyle MA, Chirmule N, Zhang Y, Wilson JM. "Stealth" adenoviruses blunt cell-mediated and humoral immune responses against the virus and allow for significant gene expression upon readministration in the lung. J Virol 2001; 75: 4792-801.

[25] Croyle MA, Chirmule N, Zhang Y, Wilson JM. PEGylation of E1deleted adenovirus vectors allows significant gene expression on readministration to liver. Hum Gene Ther 2002; 13: 1887-1900.

[26] Alemany R, Suzuki K, Curiel DT. Blood clearance rates of adenovirus type 5 in mice. J Gen Virol 2000; 81: 2605-9.

[27] Ogawara K, Rots MG, Kok RJ, et al. A novel strategy to modify adenovirus tropism and enhance transgene delivery to activated vascular endothelial cells in vitro and in vivo. Hum Gene Ther 2004; 15: 433-43.

[28] Hedley SJ, Chen J, Mountz JD, et al. Targeted and shielded adenovectors for cancer therapy. Cancer Immunol Immunother 2006; 55: 1412-9.

[29] Dmitriev IP, Kashentseva EA, Curiel DT. Engineering of adenovirus vectors containing heterologous peptide sequences in the $\mathrm{C}$ terminus of capsid protein IX. J Virol 2002; 76: 6893-9.

[30] Campos SK, Parrott MB, Barry MA. Avidin-based targeting and purification of a protein IX-modified, metabolically biotinylated adenoviral vector. Mol Ther 2004; 9: 942-54.

[31] Li J, Le L, Sibley DA, Mathis JM, Curiel DT. Genetic incorporation of HSV-1 thymidine kinase into the adenovirus protein IX for functional display on the virion. Virology 2005; 338: 247-58.

[32] Meulenbroek RA, Sargent KL, Lunde J, Jasmin BJ, Parks RJ. Use of adenovirus protein IX (pIX) to display large polypeptides on the virion--generation of fluorescent virus through the incorporation of pIX-GFP. Mol Ther 2004; 9: 617-24.

[33] Le LP, Everts M, Dmitriev IP, Davydova JG, Yamamoto M, Curiel DT. Fluorescently labeled adenovirus with pIX-EGFP for vector detection. Mol Imaging 2004; 3: 105-16. 
[34] Le LP, Le HN, Dmitriev IP, et al. Dynamic monitoring of oncolytic adenovirus in vivo by genetic capsid labeling. J Natl Cancer Inst 2006; 98: 203-14

[35] Hakkarainen T, Hemminki A, Curiel DT, Wahlfors J. A conditionally replicative adenovirus that codes for a TK-GFP fusion protein (Ad5Delta24TK-GFP) for evaluation of the potency of oncolytic virotherapy combined with molecular chemotherapy. Int J Mol Med 2006; 18: 751-9.

[36] Matthews QL, Sibley DA, Wu H, et al. Genetic incorporation of a herpes simplex virus type 1 thymidine kinase and firefly luciferase fusion into the adenovirus protein IX for functional display on the virion. Mol Imaging 2006; 5: 510-9.

[37] Peters T. All about albumin: biochemistry, genetics, and medical applications. Academic Press, San Diego 1996.

[38] Osborn BL, Sekut L, Corcoran M, et al. Albutropin: a growth hormone-albumin fusion with improved pharmacokinetics and pharmacodynamics in rats and monkeys. Eur J Pharmacol 2002; 456: 149-58.

[39] Halpern W, Riccobene TA, Agostini H, et al. Albugranin, a recombinant human granulocyte colony stimulating factor (G-CSF) genetically fused to recombinant human albumin induces prolonged myelopoietic effects in mice and monkeys. Pharm Res 2002; 19: 1720-9.

[40] Yeh P, Landais D, Lemaitre M, et al. Design of yeast-secreted albumin derivatives for human therapy: biological and antiviral properties of a serum albumin-CD4 genetic conjugate. Proc Natl Acad Sci USA 1992; 89: 1904-8.

[41] Osborn BL, Olsen HS, Nardelli B, et al. Pharmacokinetic and pharmacodynamic studies of a human serum albumin-interferonalpha fusion protein in cynomolgus monkeys. J Pharmacol Exp Ther 2002; 303: 540-8.

[42] Albuferon [http://www.hgsi.com/albuferon.html]. Rockville, MD. Human genome sciences 2008. Available from: http://www. hgsi.com

[43] Kinghorn KJ, Crowther DC, Sharp LK, et al. Neuroserpin binds Abeta and is a neuroprotective component of amyloid plaques in Alzheimer disease. J Biol Chem 2006; 281: 29268-77.

[44] Johansson MU, Frick IM, Nilsson H, et al. Structure, specificity, and mode of interaction for bacterial albumin-binding modules. $\mathbf{J}$ Biol Chem 2002; 277: 8114-20.
[45] Kraulis PJ, Jonasson P, Nygren PA, et al. The serum albuminbinding domain of streptococcal protein $\mathrm{G}$ is a three-helical bundle: a heteronuclear NMR study. FEBS Lett 1996; 378: 190-4.

[46] Vellinga J, Rabelink MJ, Cramer SJ, et al. Spacers increase the accessibility of peptide ligands linked to the carboxyl terminus of adenovirus minor capsid protein IX. J Virol 2004; 78: 3470-9.

[47] He TC, Zhou S, da Costa LT, Yu J, Kinzler KW, Vogelstein B. A simplified system for generating recombinant adenoviruses. Proc Natl Acad Sci USA 1998; 95: 2509-14.

[48] Jones N, Shenk T. Isolation of deletion and substitution mutants of adenovirus type 5. Cell 1978; 13: 181-8.

[49] Sumida SM, Truitt DM, Lemckert AA, et al. Neutralizing antibodies to adenovirus serotype 5 vaccine vectors are directed primarily against the adenovirus hexon protein. J Immunol 2005; 174: 7179-85.

[50] Kurachi S, Koizumi N, Sakurai F, et al. Characterization of capsidmodified adenovirus vectors containing heterologous peptides in the fiber knob, protein IX, or hexon. Gene Ther 2007; 14: 266-74

[51] Colby WW, Shenk T. Adenovirus type 5 virions can be assembled in vivo in the absence of detectable polypeptide IX. J Virol 1981 39: $977-80$

[52] Ghosh-Choudhury G, Haj-Ahmad Y, Graham FL. Protein IX, a minor component of the human adenovirus capsid, is essential for the packaging of full length genomes. EMBO J 1987; 6: 1733-9.

[53] Lutz P, Rosa-Calatrava M, Kedinger C. The product of the adenovirus intermediate gene IX is a transcriptional activator. J Virol 1997; 71: 5102-9.

[54] Marsh MP, Campos SK, Baker ML, Chen CY, Chiu W, Barry MA Cryoelectron microscopy of protein IX-modified adenoviruses suggests a new position for the C terminus of protein IX. J Virol 2006; 80: 11881-6.

[55] Sarkioja M, Pesonen S, Raki M, et al. Changing the adenovirus fiber for retaining gene delivery efficacy in the presence of neutralizing antibodies. Gene Ther 2008; 15: 921-9.

[56] Myhre S, Henning P, Granio O, et al. Decreased immune reactivity towards a knobless, affibody-targeted adenovirus type 5 vector. Gene Ther 2007; 14: 376-81.

(C) Hedley et al.; Licensee Bentham Open.

This is an open access article licensed under the terms of the Creative Commons Attribution Non-Commercial License (http://creativecommons.org/licenses/by$\mathrm{nc} / 3.0 /$ ) which permits unrestricted, non-commercial use, distribution and reproduction in any medium, provided the work is properly cited. 